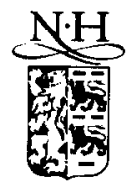

ELSEVIER

\title{
Phonon squeezing in superconducting borocarbides
}

\author{
T. Hakioğlu, V.A. Ivanov *, A.S. Shumovsky, B. Tanatar \\ Physics Department, Bilkent University, Bilkent, TR-06533, Ankara, Turkey
}

Received 26 September 1994

\begin{abstract}
The recently discovered superconductor $\mathrm{LuNi}_{2} \mathrm{~B}_{2} \mathrm{C}$ is investigated in the context of strong electron-electron correlations modulated by a squeezed phonon mode propagating in the perpendicular direction to the layers with longitudinal polarization. The squeezed phonons arise in the anharmonic lattice expansion since the linear electron-phonon interaction vanishes due to the structure of the $\mathrm{NiB}_{4}$ tetrahedra. The observed weak isotope effect and small dependence of $T_{\mathrm{c}}$ on pressure is qualitatively understood within the framework of this model.
\end{abstract}

The observation of a superconducting phase transition in the intermetalic compound $\mathrm{LuNi}_{2} \mathrm{~B}_{2} \mathrm{C}$ has been reported in a number of recent publications [ 1 $5]$. There is strong evidence to believe that these $\mathrm{Ni}$ based boride carbides are the first example of a new family of superconductors [6]. Recently calculated electronic band properties $[6,7]$ suggest that the almost filled Nid electron band $(2-3 \mathrm{eV})$ has a peak in the density of states slightly centered below $E_{\mathrm{F}}$. On the other hand in the crucial valence level photoemission measurement of the normal-state electronic structure of $\mathrm{YNi}_{2} \mathrm{~B}_{2} \mathrm{C}[8]$ the peak [6,7] was not observed in the $\mathrm{Ni}$ electron density of states because of the effects of strong electron-electron correlations. However, it was suggested that the interaction of the high-frequency boron optical $\mathrm{a}_{1 \mathrm{~g}}$ mode with the unusually broad s-p band $(30 \mathrm{eV})$ might play some role in the superconductivity of borocarbides. The $\mathrm{a}_{\mathrm{lg}}$ zone-center optical modes correspond to the vertical displacement of boron atoms with respect to the $\mathrm{Ni}$

\footnotetext{
* Corresponding author. Address: Institute for Molecular Science, Okazaki National Research Institutes Center, Myodaiji, Okazaki 444, Japan.
}

planes. It should be noted that the presence of the transition metal is the key to the important role of the electron-electron correlations in the formation of the superconducting state. On the other hand, the layered crystalline structure of this new type shows the possible contribution of transverse phonons as also implied by the boron isotope effect measurements [9]. In fact several band features with respect to their position around the $E_{\mathrm{F}}$ are found to be extremely sensitive to the $\mathrm{NiB}_{4}$ tetragonal geometry. Therefore, in the present publication we would like to note the main peculiarities of electron-phonon coupling in borocarbides of the general class $(\mathrm{LnC})_{l}(\mathrm{NiB})_{m}$.

The crystal structure [5] consists of the squareplanar Ni layers sandwiched between the B planes. The boron atoms form the tetrahedra together with the nickel atoms and $\mathrm{Ni}-\mathrm{B}$ shear is sandwiched between the $\mathrm{Lu}-\mathrm{C}$ planes. The $\mathrm{a}_{1 \mathrm{~g}}$ phonons contribute via deformations of the tetragonal $\mathrm{NiB}_{4}$ bond angles and in the planes they modulate the hopping amplitude through second-order effects. Due to the strong $\mathrm{Ni}-\mathrm{B}$ coupling small shifts in the $\mathrm{Ni}$ atomic equilibrium positions can be separated into displacement in the layers and displacement perpendicular to the lay- 
ers. Then it follows from a simple geometric consideration that [10]

$r_{i j}=r_{0}+u_{\|}+\frac{1}{2 r_{0}} u_{\perp}^{2}+O\left(u^{3}\right)$,

where $r_{i j}$ is the distance between two neighbors in the Ni square planes, $r_{0}$ is the lattice constant $\left(r_{0} \simeq 2.45\right.$ $\AA$ [5] ), $u_{\|}$and $u_{\perp}$ are the displacements in the plane and in the perpendicular directions, respectively. The expansion of the hopping integral with respect to small $u_{\|}$and $u_{\perp}$ is,

$t_{i j}\left(r_{i j}\right)=t_{i j}^{0}-\beta_{i j}\left(u_{i}+\frac{1}{2 r_{0}} u_{\perp}^{2}\right)$,

where $t_{i j}^{0}=t_{i j}\left(r_{0}\right)$ and $\beta>0$ is the absolute value of the first derivative of $t_{i j}$ with respect to the lattice constant. Because of the isotropy in the Ni layer the linear term in Eq. (2) is negligible in comparison with the bilinear term in $u_{\perp}$. Therefore the phonon contribution in the lowest order is represented by the square of the displacement in the transverse direction to the Ni layers. This type of higher-order electron-phonon interaction can lead to an effective electron-electron interaction produced by the phonon exchange. In transition-metal superconductors the influence of the e-e interactions on the electron-phonon interaction and visa versa is more important than that in conventional metals with high screening [11,12]. There the lattice vibrations lead to the variations of tunneling amplitude $t$ and site energies $\epsilon$ [13] as

$$
\begin{aligned}
& \delta t=t-t^{0}=-\frac{t}{r_{0}} u_{i j}, \\
& \delta \epsilon=\epsilon-\epsilon^{0}=-\gamma U\left(\frac{u_{i j}}{r_{0}}\right)^{2},
\end{aligned}
$$

where $u_{i j}$ describes the relative displacement of ions between neighboring sites $i, j$ and $\gamma \sim 1$. Considering that $u_{\perp} / r_{0} \sim 0.1$, our electron-phonon contribution to the site energies $\delta \epsilon_{i} \sim 10^{-2} U \simeq 0.1 \mathrm{eV}$ for Coulomb interaction $U \sim 10 \mathrm{eV}$ (according to Ref. [8] the lower threshold value is about $U \sim 5 \mathrm{eV}$ ) and $\delta t \sim 0.1 t$ is essential in calculating the ground-state properties. For non-degenerate electrons of one kind the hamiltonian of this phonon-assisted interaction can be written as

$$
\begin{aligned}
\mathscr{H} & =\sum_{q, \lambda} \omega_{q, \lambda} b_{q, \lambda}^{\dagger} b_{q, \lambda} \\
& +\sum_{\langle i, j\rangle, \nu, \sigma} n_{i, \sigma}^{\nu}\left\{\epsilon^{0}-\frac{U}{r_{0}^{2}} \sum_{q, q^{\prime}, \lambda, \lambda^{\prime}} D_{q, \lambda^{\prime} \lambda^{\prime}}^{q^{\prime}}\left(b_{q, \lambda}^{\dagger}+b_{-q, \lambda}\right)\right. \\
& \left.\times\left(b_{q^{\prime}, \lambda^{\prime}}^{\dagger}+b_{-q^{\prime}, \lambda^{\prime}}\right) \delta_{q,-q^{\prime}} \delta_{\lambda, \lambda^{\prime}}\right\} \\
& +\sum_{\langle i, j\rangle, \nu, \sigma} c_{i, \sigma}^{\dagger} c_{j, \sigma}\left\{t^{0}-\frac{\beta}{2 r_{0}} \sum_{q, q^{\prime}, \lambda, \lambda^{\prime}} D_{q, \lambda^{\prime}}^{q^{\prime} \lambda^{\prime}}\left(b_{q, \lambda}^{\dagger}+b_{-q, \lambda}\right)\right. \\
& \left.\times\left(b_{q^{\prime}, \lambda^{\prime}}^{\dagger}+b_{-q^{\prime}, \lambda^{\prime}}\right) \delta_{q,-q^{\prime}} \delta_{\lambda, \lambda^{\prime}}\right\}+\mathscr{H}_{\text {intra }} .
\end{aligned}
$$

Here the delta symbols indicate the total phononmomentum conservation independently from the electron momentum since $q$ is orthogonal to the planes. All possible intra-atomic interactions of electrons in different orbitals $\nu$ are included in the last term. The operator $b_{q, \lambda}\left(b_{q, \lambda}^{\dagger}\right)$ annihilates (create) phonons with momentum $q$ in the phonon branch indicated by $\lambda$. The $\omega_{q, \lambda}$ represents the phonon energy. The $n_{i, \sigma}^{\nu}=c_{i, \sigma}^{\nu \dagger} c_{i, \sigma}^{\nu}$ is the number operator of electrons in the $\nu$ orbital with spin projection $\sigma$ at site $i$ of the Ni plane. In Eq. (4) we have

$D_{q, \lambda^{\prime}}^{q^{\prime}, \lambda^{\prime}}=\frac{1}{2 M N \sqrt{\omega_{q, \lambda} \omega_{q^{\prime}, \lambda^{\prime}}}}$,

where $N$ is the number of unit cells and $M$ is the reduced mass of the nickel and boron atoms. The ground-state eigenfunction can be chosen in the simplest form of a direct product of electron and phonon wave functions

$\left|\psi_{\mathrm{G}}\right\rangle=\left|\psi_{\mathrm{e}}\right\rangle\left|\psi_{\mathrm{ph}}\right\rangle$.

It is known in the context of narrow band polaron modes that the hamiltonian (4) supports the formation of the squeezed vacuum ground state in the form

$$
\begin{aligned}
& \left|\psi_{\mathrm{G}}\right\rangle=\prod_{q} \mathscr{P}\left(\xi_{q}\right)|0\rangle \\
& =\prod_{q} \exp \left\{\xi_{q}\left(b_{-q}^{\dagger} b_{q}^{\dagger}-b_{-q} b_{q}\right)\right\}|0\rangle,
\end{aligned}
$$

with $\xi_{q}$ being the variational squeezing parameter. This new squeezed phonon state has first been applied to condensed matter physics in the intermediate valence problem $[14,15]$, bipolaron superconductivity [16] and the superconductivity in the 
Hubbard model [17]. In Refs. [16] and [17] the variational procedure was applied to calculate the superconducting state induced by the e-ph interaction. In our model due to the nature of the e-ph interaction the electron momentum is not coupled to the phonon momentum. This indicates that the phonons are not of conventional exchange type, therefore the ground state of the electrons is not of BCS but of metallic type. We will study the superconductivity in our suggested borocarbide model caused by the interaction of d electrons. In conventional superconductors due to the large coherence length only properties averaged over a large number of unit cells are important. In new superconductors, however, the coherence length is typically defined by few $(\sim 10-30)$ unit cells and the complexity of different branches in the phonon spectrum with different ion masses is very high. Here we consider the simplest and natural starting point and assume only the phonon branches transverse to the Ni planes with an averaged dispersion given by

$\frac{1}{\omega^{2}}=\sum_{\lambda} \frac{1}{\omega_{\lambda}^{2}}$

In our one-parameter variational ground state, the ground-state energy $E_{\mathrm{g}}$ is calculated by minimizing

$E_{\mathrm{g}}=\left\langle\psi_{\mathrm{e}}\left|\left\langle\psi_{\mathrm{ph}}|\mathscr{H}| \psi_{\mathrm{ph}}\right\rangle\right| \psi_{\mathrm{e}}\right\rangle$

with respect to the squeezing parameter $\xi_{q}$. With the help of the relation

$$
\mathscr{S}^{\dagger}\left(\xi_{q}\right) b_{q} \mathscr{S}\left(\xi_{q}\right)=c_{q} b_{q}+s_{q} b_{-q}^{\dagger},
$$

where $c_{q}=\cosh 2 \xi_{q}$ and $s_{q}=\sinh 2 \xi_{q}$, we first calculate the effective hamiltonian with one orbital electrons (dropping the index $\nu$ ),

$$
\begin{aligned}
\mathscr{H}_{\mathrm{e}} & =\left\langle\psi_{\mathrm{ph}}|\mathscr{H}| \psi_{\mathrm{ph}}\right\rangle \\
& =\sum_{i j\rangle, \sigma} n_{i, \sigma}\left\{\epsilon^{0}-\frac{U}{r_{0}^{2}} \sum_{q} D_{q}\left(c_{q}+s_{q}\right)^{2}\right\} \\
& +\sum_{i, \sigma} c_{i \sigma}^{\dagger} c_{j \sigma}\left\{t^{0}-\frac{\beta}{2 r_{0}} \sum_{q} D_{q}\left(c_{q}+s_{q}\right)^{2}\right\} \\
& +\sum_{q} \omega_{q} s_{q}^{2}+\mathscr{H}_{\text {intra }} .
\end{aligned}
$$

Then using Eqs. (9) and (11), $E_{\mathrm{g}}$ is found to be minimum for $\mathrm{e}^{-4 \xi q}=\left\{1-4 \kappa D_{q} / \omega_{q}\right\}^{1 / 2}$ and $\kappa=\frac{\beta}{2 r_{0}}\left\langle\sum_{\langle i j\rangle, \sigma} c_{i, \sigma}^{\dagger} c_{j, \sigma}\right\rangle+\frac{U}{r_{0}^{2}}\left\langle\sum_{i, \sigma} n_{i, \sigma}\right\rangle$

The expectation values in Eq. (12) are taken in the metallic ground state. The renormalization of the hopping amplitude $t_{i j}^{0}$ and the site energy $\epsilon_{i}^{0}$ are finally found as

$$
\begin{aligned}
& \delta t=-\frac{\beta}{2 r_{0} \kappa} \sum_{q} \frac{\eta}{\omega_{q}} \frac{1}{\left(1-\frac{4 \eta}{\omega_{q}^{2}}\right)}, \\
& \delta \epsilon=-\frac{U}{r_{0}^{2} \kappa} \sum_{q} \frac{\eta}{\omega_{q}} \frac{1}{\left(1-\frac{4 \eta}{\omega_{q}^{2}}\right)},
\end{aligned}
$$

where $\eta / \omega_{q}=\left(\kappa / 2 M N \omega_{q}\right)$ describes the energy scale for the anharmonic e-ph coupling. In the calculation of Eqs. (13) we consider the phonon density of states $\rho(\omega)$ as a one-peak broadened function which smoothly vanishes for $\omega \leq 2 \sqrt{\eta}$ and for $\omega \geq \omega_{0}$. In Fig. 1 (a) the first of Eqs. (13) ( $\delta t$ as a function of $\eta / \omega_{\mathrm{D}}^{2}$ ) is given. Fig. 1(b) is the shift in the ground state energy $\Delta E_{\mathrm{g}}$ described by the full electron-phonon part of the hamiltonian (11) with squeezed coupling from that without, as a function of the squeezed coupling $\eta / \omega_{\mathrm{D}}^{2}$. It can be seen that for $0 \leq \eta / \omega_{\mathrm{D}}^{2} \leq 0.08$ the

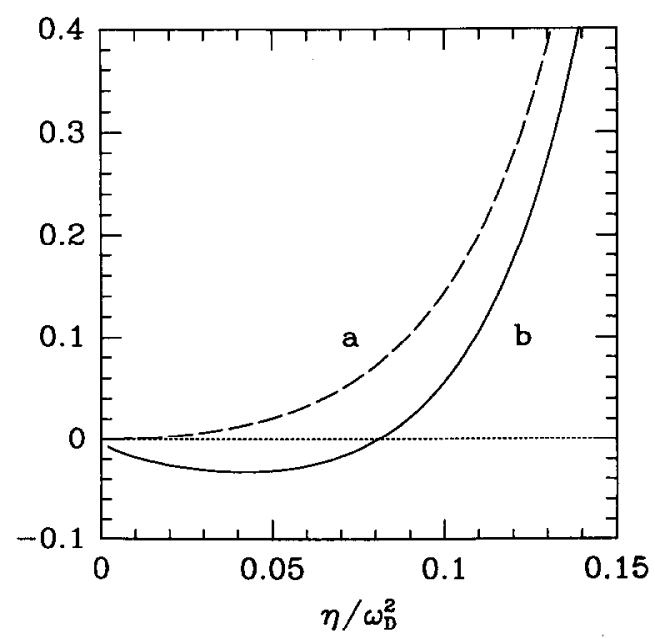

Fig. 1. (a) Renormalization of the hopping amplitude $\delta t$ as a function of the squeezed coupling constant $\eta / \omega_{\mathrm{D}}^{2}$; (b) The shift in the ground-state energy $\Delta E_{\mathrm{g}}$ of the electron-phonon part of the hamiltonian (11) with squeezed coupling from that without a function of the squeezed coupling constant $\eta / \omega_{D}^{2}$ 
squeezed phonon ground state is energetically favoured. The minimum of $E_{\mathrm{g}}$ in Fig. 1 corresponds to $\eta / \omega_{\mathrm{D}}^{2} \sim 0.04-0.05$.

Because of the partially filled $\mathrm{d}$ shell of the $\mathrm{Ni}$ ion embedded in the compound $(\mathrm{LnC})_{l}(\mathrm{NiB})_{m}$, strong $\mathrm{d}-\mathrm{d}$ correlations are expected to be important in the planes. In the layered superconducting compound $\mathrm{La}_{2-x} \mathrm{Sr}_{x} \mathrm{NiO}_{4}$ the octahedral oxygen surrounding leads to splitting of the electron energy in the completely filled lower $t_{2 g}$ levels and partially filled upper $\mathrm{e}_{\mathrm{g}}$ levels where diamagnetic anomalies are observed $[18,19]$. In borocarbides tetrahedral boron surrounding [5] leads to completely filled lower $\mathrm{e}_{\mathrm{g}}$ levels and partially filled upper $t_{2 \mathrm{~g}}$ levels with electron wave function of different angular symmetry $x y, y z$, $x z$. Therefore the effective electron hamiltonian of our problem is more complicated than Eq. (11). Because of the ideal boron surrounding in borocarbides, let us assume three-fold orbital degeneracy of $\mathrm{Ni}$ electrons participating in the superconductivity (contrary to the two-fold $\mathrm{e}_{\mathrm{g}}$ degeneracy in nickelates [20]). The basis functions of electrons will be classified corresponding to the irreducible representation of $t_{2 \mathrm{~g}}$ : $a=(x y), b=(y z), c=(z x)$. Let us take for the vacuum statc the half filled shell $t_{2 \mathrm{~g}}^{3}$ of ion $\mathrm{M}(\mathrm{d} 7)=\mathrm{M}\left(\mathrm{e}_{\mathrm{g}}^{4} \mathrm{t}_{2 \mathrm{~g}}^{3}\right)=\mathrm{Ni}^{3+}, \mathrm{Pd}^{3+}, \ldots$. Then the quartet ground state can be expressed as (four triplets)

$$
\begin{aligned}
|\sigma \sigma \sigma\rangle & =a_{\sigma}^{\dagger} b_{\sigma}^{\dagger} c_{\sigma}^{\dagger}|0\rangle, \quad S_{z}=3 \sigma / 2, \sigma= \pm 1, \\
\left|T_{\sigma}\right\rangle & =\frac{1}{\sqrt{3}}\left(a_{\bar{\sigma}}^{\dagger} b_{\sigma}^{\dagger} c_{\sigma}^{\dagger}+a_{\sigma}^{\dagger} b_{\bar{\sigma}}^{\dagger} c_{\sigma}^{\dagger}+a_{\sigma}^{\dagger} b_{\sigma}^{\dagger} c_{\sigma}^{\dagger}\right)|0\rangle, \\
S_{z} & =\sigma / 2 .
\end{aligned}
$$

In the tight-binding method of correlated electrons $[21,22]$ the general intraatomic hamiltonian can be diagonalized in terms of triplets (14), "quadruplets" $(2--),(2++),\left(2_{a} T_{b c}\right)$ where $2_{a}=\sigma a_{a}^{\dagger} a_{\sigma}^{+}|0\rangle$ is the Hubbard "doublet" of the $a$ orbital and $T_{b c}=\frac{1}{2} \sqrt{2}\left(b_{\sigma}^{\dagger} c_{\bar{\sigma}}^{+}+b_{\bar{\sigma}}^{\dagger} c_{\sigma}^{\dagger}\right)$ is a triplet state with $S_{z}=0$ "doublets" etc. The corresponding eigenenergy levels $\epsilon_{p}$ of $\mathscr{H}_{\text {intra }}$ include all essential intraatomic interactions (Hubbard, Coulomb, Hund). In terms of these eigenenergies we have $\mathscr{H}_{\text {intra }}=\Sigma_{p} \epsilon_{p} X_{p}^{p}$ the generalized Hubbard-Okubo $X$ operators [23] $X_{s}^{p}=X_{\gamma}(p$, $s)$ project the energy state $p$ into $s$ differing by one electron configuration. The correlated energy bands are formed due to the itinerary character of the intraatomic transitions.
In the electronic structure of borocarbides the upper correlated band of $t_{2 \mathrm{~g}}$ electrons (involving the eighth $\mathrm{d}$ electron of $\mathrm{Ni}^{3+/ 2+}$ ion) is formed due to the transitions from polar "quadruplets" to ground state "triplets". The expansion of the one-electron annihilation operator in terms of the orthogonal $X$ operators is determined by the genealogical coefficients $g_{\gamma}[23]$

$$
\begin{aligned}
a_{\sigma} & =\sum_{\gamma} g_{\gamma} X_{\gamma}(p, s) \\
& =\frac{\sigma}{\sqrt{3}} X_{T_{\tilde{\sigma}}}^{2 \sigma \sigma}+\sqrt{2 / 3} \sigma X_{T_{\bar{\sigma}}}^{2 a T_{b c}}+\sigma X_{\bar{\sigma} \bar{\sigma}}^{2 \sigma \bar{\sigma}}+\ldots
\end{aligned}
$$

and similarly for $b_{\sigma}$ and $c_{\sigma}$ by cyclic permutation of the indices. In the presence of strong intraatomic d$d$ correlations the effective hamiltonian describing the normal phase of borocarbides is

$$
\mathscr{H}=\sum_{\gamma,\langle i, j\rangle, a} g_{\gamma}^{a} g_{\gamma}^{a} X_{i}^{\gamma} X_{j}^{\gamma}-\mu \sum_{i} n_{i}
$$

where $t_{a a}^{0}$ is given by Eq. (13) and $\mu$ is the chemical potential which includes the effect of narrowing of the energy bands due to phonon squeezing. Because of the angular symmetry of the basis functions $\psi_{x y, x z, y z}$ the interorbital hopping vanishes (i.e. $t_{a b}^{0}=t_{a c}^{0}=$ $t_{b c}^{0}=0$ ).

Here the lifetime effects due to retardation and spin flip processes $[24,25]$ are ignored. Due to the strong intraatomic correlations $(U \gg t)$ we may also confine intraatomic transitions defined in Eq. (15) forming the upper correlated band of $t_{2 g}$ electrons with single-particle energies,

$\xi_{\mathrm{p}}=f \sum_{\gamma} g_{\gamma}^{2} t_{p}-\mu=f\left(\frac{1}{3}+\frac{2}{3}+1\right) t_{p}-\mu$,

where $t_{p}=\sum_{\delta} t^{0} \mathrm{e}^{p \cdot \delta}=2 t^{0}\left(\cos p_{x} r_{0}+\cos p_{y} r_{0}\right)$ and $f=$ $n_{3}+n_{4}$ is the end factor [23] of the generalized DT, consisting of populations of the ground-state "triplet" levels $n_{3}$ and polar "quadruplet" levels $n_{4}$. Taking into account the four-fold and six-fold degeneracy of "triplets" and "quadruplets" in the expansion of Eq. (15) we obtain

$$
\begin{aligned}
& 4 n_{3}+6 n_{4}=1, \\
& 4 n_{3}+3 n_{4}=\frac{3-n}{3},
\end{aligned}
$$

where $n$ is the number of electrons in the upper correlated band of $\mathrm{Ni}^{3+/ 2+}\left(\mathrm{d}^{7+n}\right)$ plane. Therefore as- 
suming in the compound $\left(\mathrm{Ln}^{3+} \mathrm{C}^{2-}\right)_{l}\left(\mathrm{Ni}^{3-n} \mathrm{~B}^{3-}\right)_{m}$ the same distribution of charge as in Ref. [10], the charge balance requires $n=l / m$ and

$f=n_{3}+n_{4}=\frac{9+2 n}{36}$.

The equation for the chemical potential follows from

$$
\begin{aligned}
n & =3+2 f \sum_{\gamma} g_{\gamma}^{2} T \sum_{p, \omega_{\mathrm{M}}} G_{\gamma}\left(\boldsymbol{p}, \omega_{\mathrm{M}}\right) \mathrm{e}^{\mathrm{i} \omega_{\mathrm{M}} \delta} \\
& =3+\frac{3+2 n}{9} \sum_{\boldsymbol{p}} \theta\left(-\xi_{p}\right),
\end{aligned}
$$

where $\omega_{\mathrm{M}}$ are the Matsubara frequencies. For rectangular density of states $\rho(\omega)=(1 / 2 W)\left(\theta\left(W^{2}-\epsilon^{2}\right)\right.$ we get from Eq. (19)

$\frac{\mu}{W}=\frac{9-16 n}{18}$,

where the half band width $W=2 t$ and $t$ is given by Eqs. (3) and (13). Eqs. (17), (19) and (21) determine the one-particle energies $\xi_{\mathrm{p}}$, forming the upper correlated band in the normal state. With decreasing temperature, the superconducting transition is found from the self-consistent solution of the homogeneous Bethe-Salpeter vertex as the (kinematical superconductivity $[23,24])$

$1=\sum_{p} \frac{t_{p}}{\xi_{\mathrm{p}}} \tanh \frac{\xi_{\mathrm{p}}}{2 T_{c}}$

In the logarithmic approximation the solution is described by

$T_{\mathrm{c}}=\frac{W}{3} \sqrt{7 n(1-n)} \exp \left\{-\frac{\left(\frac{3}{4}\right)^{4}}{\frac{9}{16}-n}\right\}$

The superconductivity exists for $n=l / m<\frac{9}{16}$ (i.e. superconducting compound with chemical formula $\left.\operatorname{LuCNi}_{2}\left(3 d^{7}\right) B_{2}\right)$. In agreement with the baric measurements we get an only $\mathrm{d} T_{\mathrm{c}} / \mathrm{d} p$ in agreement with the experiment [2]. The unusual isotope effect [9] can also be explained by the boron mass dependence of the prefactor in Eq. (23).

The renormalized half band width $W$ can be computed from Eqs. (12) and (13). The boron isotope effect can be found from the mass dependence of the dimensionless parameter $\eta / \omega_{\mathrm{D}}^{2}$. In Fig. (1(a)) $\delta t$ is shown as a function of the dimensionless ratio $u=\eta$ / $\omega_{\mathrm{D}}^{2}$. Due to the large band width, we have $\omega_{\mathrm{D}} / t^{0} \ll 1$.

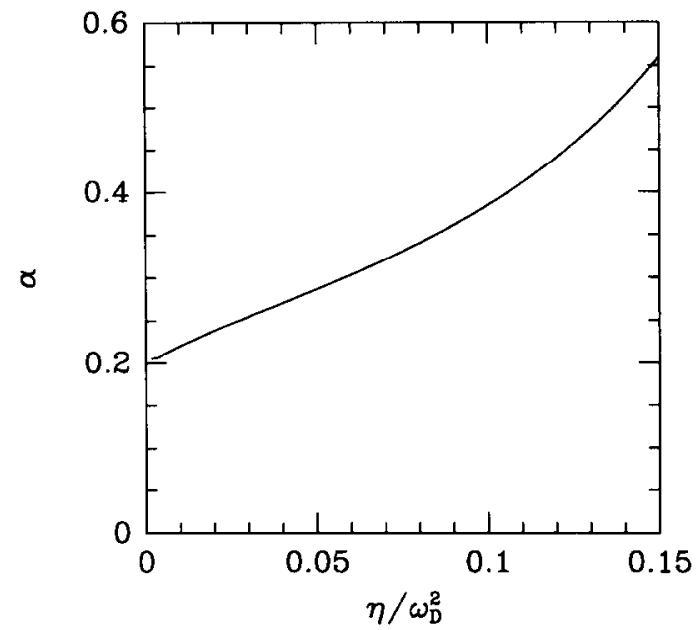

Fig. 2. The boron isotope exponent $\alpha_{\mathrm{B}}$ as a function of the squeezed coupling constant $\eta / \omega_{\mathrm{D}}^{2}$.

If we also reasonably assume $\beta \propto M^{-\nu}$ one finds that the boron isotope exponent, $\alpha_{\mathrm{B}}=-\partial \ln T_{\mathrm{c}} / \partial \ln M$ can be approximately written as

$\alpha_{\mathrm{B}} \simeq M \frac{\partial\left(\delta t / t^{0}\right)}{\partial M}=\frac{1}{2} \frac{\delta t}{t^{0}}-\nu \frac{\omega_{\mathrm{D}}}{t^{0}} \frac{\partial}{\partial \ln u}\left(\frac{\delta t}{\omega_{\mathrm{D}}}\right)$.

In Eq. (24) the last term can be calculated from the Fig. 1. Since $\delta t / t^{0} \sim 0.1$, and the derivative of $\delta t / \omega_{\mathrm{D}}$ with respect to $u$ is negative, $\alpha_{B}$ has a positive but small value. The isotope exponent as a function of the dimensionless squeezing parameter $\eta / \omega_{\mathrm{D}}^{2}$ is presented in the Fig. 2 for $\nu=1$. At the value of the squeezing parameter where the ground-state energy is minimum (i.e. $\eta / \omega_{\mathrm{D}}^{2} \sim 0.04-0.05$ ) the isotope exponent yields $\alpha_{\mathrm{B}} \sim 0.25-0.30$ which is very close to the experimental value $\alpha_{\mathrm{B}} \sim 0.27$ [9].

\section{Acknowledgement}

This work and VI were supported by the Ministry of Education, Science and Culture of Japan, especially by the "New Program".

\section{References}

[1 ] C. Mazumdar, R. Nagarajan, G. Godart et al., Solid State Commun. 87 ( 1993$) 413$. 
[2] R. Nagarajan et al., Phys. Rev. Lett. 72 (1994) 274.

[3] R.J. Cava et al., Nature (London) 367 (1994) 146.

[4] R.J. Cava et al., Nature (London) 367 (1994) 252.

[5] T. Siegrist, H.W. Zandbergen et al., Nature (London) 367 (1994) 254.

[6] L.F. Mattheiss, T. Siegrist and R.J. Cava, Solid State Commun. 91 (1994) 587.

[7] L.F. Mattheiss, Phys. Rev. B 49 (1994) 13279.

[8] M.S. Golden, M. Knupfer, M. Kielwein et al., Europhys. Lett. (1994), to be published.

[9] D.D. Lawrie and J.P. Franck, to be published in Physica C: Proceedings of $\mathrm{M}^{2} \mathrm{~S}-\mathrm{HTSC}-\mathrm{IV}$, Grenoble, France, ed. P. Wyder (Elsevier 1994).

[10] T. Hakioḡlu, V.A. Ivanov, A.S. Shumovsky and B. Tanatar, to be published in Physica C: Proceedings of $\mathrm{M}^{2} \mathrm{~S}-\mathrm{HTSC}$ IV, Grenoble, France, ed. P. Wyder (Elsevier 1994).

[11] S. Ishihara, T. Egami and M. Tackiki, Phys. Rev. B 49 (1994) 8944.

[12] M.L. Kulić and R. Zcyher, Phys. Rev. B 49 (1994) 4395.

[13] N.M. Plakida, in: Electron-Phonon Interaction in Oxide Superconductors, Proc. First CINVESTAV Superconductivity Symp., ed. R. Baquero (World Scientific, Singapore, 1991).
[ 14] H. Zheng and K. Nasu, Phys. Rev. B 45 (1992) 2148.

[15] H. Zheng, Phys. Rev. B 38 (1988) 11865.

[16] B. Chakraverty, D. Feinberg, Z. Hang and M. Avignon, Solid State Commun. 64 (1987) 1147.

[17] M. Hiratsuka, T. Sota and H. Suzuki, Proc. Ann. Meeting of Phys. Soc. Jpn., Fukuoka, 1994, vol. 3, p. 330.

[18] Z. Kakol, J. Spalek and J.M. Honig, J. Solid State Chem. 79 (1989) 288

[19] J. Acrivos, J. Solid State Chem. (1994), to be published.

[20] V.A. Ivanov, Sov. J. Inorg. Chem. 34 (1989) 3201.

[21] V.A. Ivanov, in: Studies of High Temperature Superconductors, vol. 11, ed. A. Narlikar (Nova Science, New York, 1993).

[22] V.A. Ivanov, J. Phys. Condens. Matter 6 (1994) 2065; Physica B 186 (1993) 921.

[23] A. Bedyaev, V.A. Ivanov and V.E. Shilov, Theor. Math. Phys. 64 (1985) 163.

[24] V.A. Ivanov, Physica C 162 (1989) 1485.

[25] V.A. Ivanov and R.O. Zaitsev, Int. J. Mod. Phys. B 3 (1989) 1403.

[26] H. Takagi, H. Eisaki, S. Uchida et al., in: Proc. Ann. Meeting of Phys. Soc. Jpn., Fukuoka, 1994, vol. 2, p. 417. 